\title{
INVESTIGATION OF SUSTAINABLE URBAN DEVELOPMENT DIRECTION USING GEOGRAPHIC INFORMATION SYSTEMS (CASE STUDY: ZANJAN CITY)
}

\author{
Z. Masoumi ${ }^{1, *}$, J.V. Genderen ${ }^{2}$ \\ ${ }^{1}$ Institute for Advanced Studies in Basic Sciences, Department of Earth Science, z.masoumi@iasbs.ac.ir \\ ${ }^{2}$ Department of Earth Observation Sciences (ITC), University of Tewnte, Enschede, The Netherlands, genderen@alumni.itc.nl
}

Commission IV, WG IV/10

KEY WORDS: Sustainable urban development, GIS, TOPSIS, MCDM.

\begin{abstract}
:
The ever-increasing development of the cities and the growth of population have led to an uncontrollable sprawl of spatial structure of the cities. Hence, sustainable development and organizing of land-use planning are always an important concern of decision makers. Zanjan city, due to the existence of physical limitations and environmental issues, needs precise planning regarding city development. The aim of this study is to determine the sustainable development direction of urban development while taking the social, economic, and environmental perspectives into account. To determine suitable lands for future developments, totally 10 criteria in three groups, specifically social, economic and environmental were employed. In order to analyse the considered criteria in this study, experts' opinions were gathered to be used in determining the weights of the criteria using the analytical hierarchical process (AHP). Then, the yielded weights were applied to the criteria in a geographical information system (GIS) through spatial analyses. Finally, the technique for order of preference by similarity to ideal solution (TOPSIS), as a multi-attribute decision making (MADM) method, was utilized to extract the priority of suitable lands for city development. The results show that the most suitable lands for the purpose of future developments are located in the north-eastern and eastern parts, after which are the lands located in the north-western parts. In addition, 15 percent of city development since 2005 has occurred in unsuitable lands. Moreover, to evaluate the results, TOPSIS outcomes were compared with the weighted sum model (WSM) approach.
\end{abstract}

\section{INTRODUCTION}

In the modern world, human activities are progressing rapidly. This makes the physical development of cities a dynamic and continuous process (Delden and Hurkens, 2011). On the other hand, the growth of the urban population and the increase in urban migration have led to unsustainable construction and changes in the spatial structure of the cities. If this is a fast and unplanned process, it will lead to an inappropriate physical structure of urban spaces, and as a result will cause many urban problems (Abdullahi et al., 2015).

Suitability analysis has always been one of the most important challenges in land use planning. Since 1990, MCDMs based on GIS have attracted the attention of decision makers and urban planners in addressing location-based problems such as suitability analysis (Phua and Minowa, 2007). These techniques are classified into two categories: multi-criteria analyses (MCA) and multi-objective decision analyses (MODA). MCA are suitable tools for multi-criteria problems where the number of criteria is low and predefined. On the other hand, MODA are used when the criteria are diverse and numerous, and their priorities are important (Ullah and Mansourian, 2016).

Many studies have been done in this regard, each of which has tried to provide practical solutions to the issues of city development. Bathrellos et al. (2011), using GIS and analytical hierarchical process (AHP) model, have studied the suitable areas for the urban growth in three Greek cities with regard to natural hazards. The employed method was the weighted sum of factors. The results show that there is no consistency between the direction of growth of the studied cities and the suitable areas. The authors believed that it was due to the economic and social factors that have so significant effect on the growth of the studied cities (Bathrellos et al., 2011). Narayan and Shelton (2012), in a study in Amsterdam, have studied suitable locations in the surrounding water areas for urban growth over the next 20 years. Researchers have used satellite imagery and multicriteria evaluation models along with experts' views to locate
Urban land use planning and land administration play a very important role in the development of sustainable development. With a brief look at the important factors in urban planning and development, it can be seen that this is a multi-criteria decisionmaking problem and most of the criteria have spatial properties. Therefore, for the management of this amount of spatial and attribute data, geospatial information systems (GIS) along with multi criteria decision making (MCDM) are remarkably applicable (Shen et al., 2011).

and anticipate this development. Effat and Hegazy (2013) have used three categories of social, environmental and economic criteria in 2013 to find potential lands for development in Sinai, Egypt. Satellite imagery has been one of the most important sources of spatial information in this research. On the other hand, the weights of the layers have been obtained using AHP and the criteria have been combined with aggregated weighting method. The results show the suitable lands for urban development classified in three priority levels. Ullah and Mansourian (2016) have conducted a study in 2016 to find the suitability of lands in the vicinity of Dhaka for urban development. In this research, AHP method is used to determine weights for physical criteria, land use, facilities and urban services. In total, 14 criteria have been introduced into the model, and some constraints have been defined for urban development. Finally, MCDM method has been used to combine the criteria, and to obtain a final suitability map. The resulting map includes areas with potential to urban development. Santosh et al. (2018), using AHP method and a weighted sum approach in GIS has adopted a site suitability analysis to find appropriated areas for urban development in Chikodi taluk, Karnataka, India. In this study six thematic layers were considered such as slope, land-use/land-cover, road proximity, land value, lineaments and aspect. The final site suitability map was divided into five different suitability categories.

\footnotetext{
* Corresponding author
} 
According to the above literatures, this research attempts to investigate the role of social and economic factors, in addition paper, the TOPSIS model has been used in determining the priority of lands for future developments of the city. TOPSIS is an approach to identify an alternative which is closest to the ideal solution and farthest to the negative ideal solution in a multi-dimensional computing space (Qin et al., 2008). It has numerous advantages. It has a simple process. It is easy to use and programmable. The number of steps remains the same regardless of the number of attributes and problem size (Ic, 2012).

\section{THEORETICAL FOUNDATIONS}

In this section, the basic concepts of employed methodology have been discused.

\subsection{Sustainable urban development}

By the turn of the 1960s, the concept of development was generally used in the sense of economic development. But from the 1960s onwards, fundamental changes have emerged in the concept of development, which have greatly affected planning objectives and methods. Sustainable growth requires an evolution in the way urban areas carry out their activities such as resource use and the movement of people and goods. The physical infrastructure in addition to social and economic processes must evolve to acknowledge the challenges of growth. Sustainable urban development has been defined as development that meets the needs of the present without compromising the ability of future generations to meet their own needs. However, sustainable urban development implies a process by which sustainability can be attained, emphasizing improvement, progress and positive change, incorporating both environmental and social dimensions (Teriman et al., 2011). Therefore, urban development is not limited to the physical development of the city and should consider all the factors affecting development in the general sense. Moreover, the issue of spatial organization of urban development is very important in this regard.

Allocation is a process that analyses the capabilities of space from various aspects such as environmental, social, and economic for specific applications (Masoumi et al., 2017). The criteria used in location finding are different with respect of the type of application, but all of them are to select the suitable location. The use of these criteria requires correct and complete information of the region, and analysing information requires extensive and comprehensive research. After evaluation of the criteria, decision-making becomes possible.

\subsection{MCDM}

MCDM is concerned with solving decision and planning problems involving multiple criteria. The purpose is to support decision-makers facing such problems. Typically, there does not exist a unique optimal solution for such problems and it is necessary to use decision-maker's preferences to differentiate between solutions. There are three steps in utilizing any decision making technique as below (Triantaphyllou, 2010):

- Determining the relevant criteria

- Attach numerical measures to the relative importance of the criteria

- Process the numerical values to determine a ranking of each alternative.

Here, in this research we used TOPSIS and compare its results with commonly used weighted sum model (WSM) to evaluate the results.

\subsection{Analytical Hierarchical Process (AHP)}

The hierarchy analysis process developed by Saaty and Vargas (2012) is one of the most comprehensive approaches designed for decision making with multiple criteria. The basis of the AHP to environmental and physical factors, in determining the optimal direction of urban development for Zanjan. In this approach is on the paired comparison of decision alternatives and criteria, and is applied based on three principles: creation of a hierarchical structure, conducting paired comparison of the elements in the hierarchical structure, and determination of weights of criteria. Since the values of the paired comparisons are determined through experts' opinions, in some cases the priorities of different individuals are contradictory and the dependence of this method on analyst opinions may cause distortion and bias in the calculations. In order to resolve this issue, a unique numerical index, called compatibility index, is defined to verify the robustness of the paired comparisons matrix, the value of which should not be greater than 0.1 . The compatibility index is calculated by dividing the compatibility criterion into the mean of compatibility criterion.

\subsection{The TOPSIS mode}

MCDMs designed for complex decision-making, are divided into two main categories: multi-objective decision-making models (MODMs) and multi-attribute decision-making models (MADMs). MODMs are used for design purposes, while MADMs are used to select the best alternative. Since, the purpose of this paper is to find suitable locations among different areas, MADMs have been employed.

The TOPSIS model is one of the best MADMs which have been used extensively. In this method, $m$ options are evaluated by $n$ criteria, and the options are then ranked according to the similarity to the ideal solution. The basis of this technique is on the notion that the chosen alternative should have the least distance with the ideal solution (the best solution) and the greatest distance with the negative solution (the worst solution).

\section{METHODOLOGY}

In this section we will introduce the study area and the proposed modeling method in the research. In Figure 1, the framework of the research method is presented and in the following sections each step will be examined in detail.

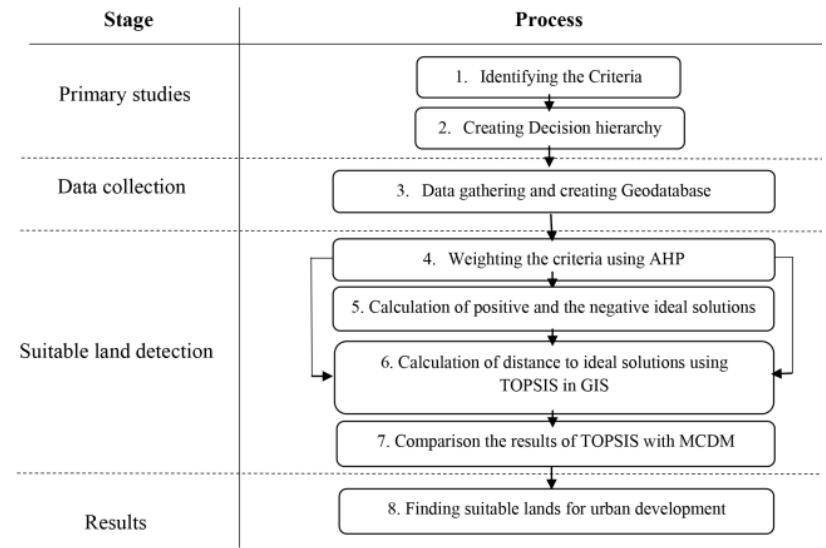

Figure 1. The proposed framework of the research method 3.1. The study area

The city of Zanjan is located in the eastern part of Zanjan province, at an average altitude of 1663 meters above sea level. The city is bounded in mountainous elevations from north, northeast, south, and southwest. The area of the city is 2,469 hectares, representing $17 \%$ of the province's total area. Natural growth of the population, the regional role of Zanjan, the development of the industry, the construction of some industries such as lead and zinc melting factories, and the expansion of administrative, educational and cultural services, have caused the immigration role of the city to be highlighted and affected the physical development of Zanjan. As a result of the 
development of the city, some agricultural lands around the city are under excessive and unregulated constructions. On the other hand, due to the lack of respect to the principles of urban planning, the costs of using infrastructures and services have increased. Figure 2 shows the location of Zanjan city in Iran.

3.2. Preparing the required layers (criteria and constraints) Criteria of land suitability for urban development are derived from multi-disciplinary scientific theories related to physical, socio-economical, and environmental attributes. All factors for evaluation of land-use suitability fall within two categories, namely the opportunities and constraints (see Zong et al., 2007). Suitability analysis essentially involves identification of
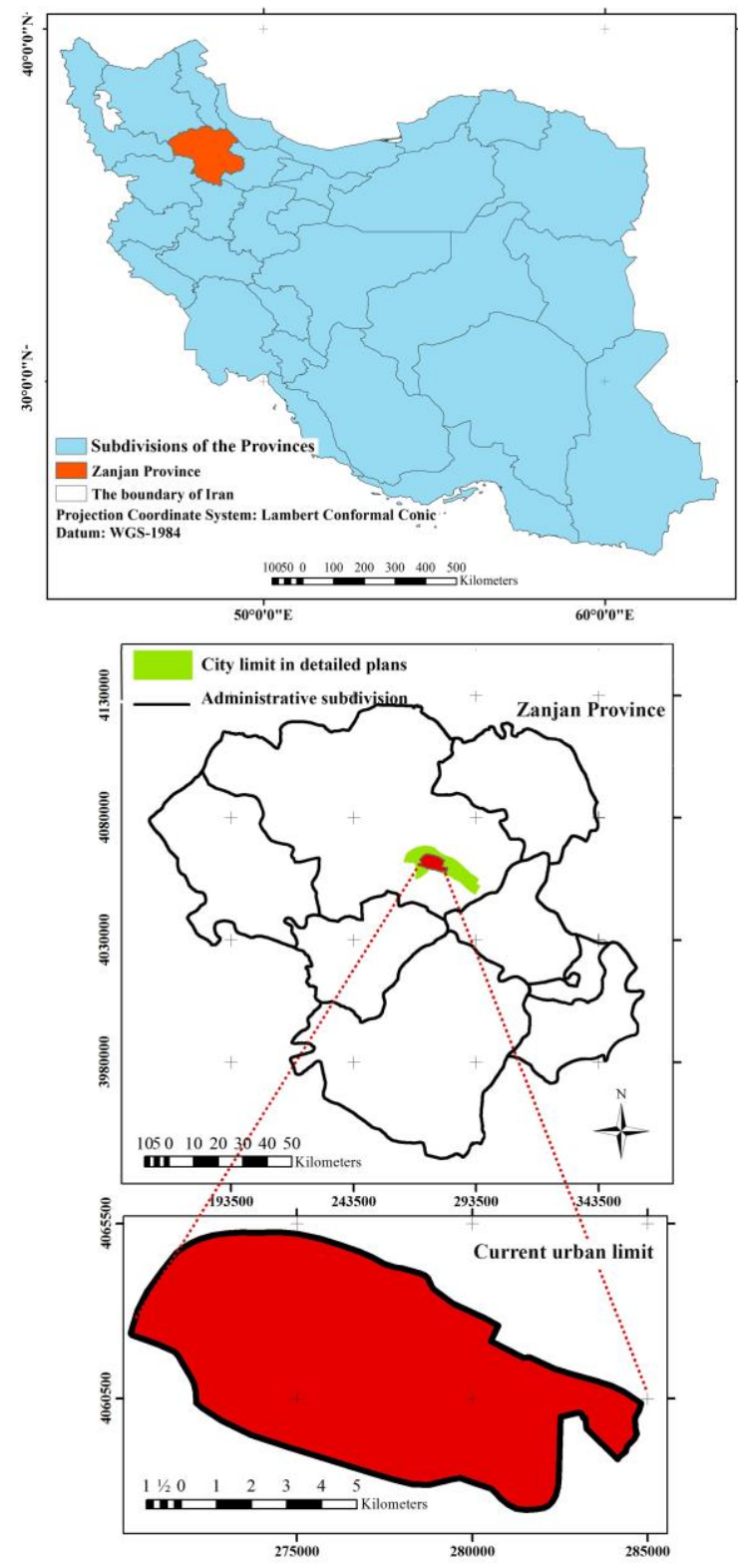

Figure 2. The geographical location of Zanjan city

opportunities and constraints for prescribed land-use(s) in a city (Liu, et al., 2014). In this research the criteria regarding the studying of suitable lands to urban land-use were extracted through the use of experts' knowledge and research references (Santosh et al., 2018; Martinat et al., 2016; Ullah and Mansourian, 2016; Abdullahi et al., 2015; Liu, et al., 2014).

The conditions used in this paper are shown in Table 1. Also, in Table 1 , the rating of each of the quantitative sub-criteria and their corresponding classifications are given. In Table 1, Class 1 indicates the best value and Class 4 shows unsuitable values. To extract the values of boundaries (thresholds) for classification of each factors, at first, we used the literature reviews. But we concluded that every literature, based on the special situations on study area, has its own categories. For example, see Liu et al., 2014, Abdullahi, 2015, Sontash et al., 2018. Also, they have been used different factors and classifications. Then, we decide to use the knowledge of the experts in Municipality of Zanjan city, Plan and Budget Organization and Ministry of roads and Urban development branch of Zanjan city. To be more coordinated, we wanted experts to classify the values of each factor in 4 classes (Not suitable, Low suitable, Medium Suitable and High suitable). It should be stated that we used 10 experts' knowledge to summarize the issue. Finally, using Delphi method and three times revisions of experts' knowledge, we summarized the ideas about classification and thresholds. In order to apply the constraints, sites that do not have the conditions are removed from the search space of the problem. The spatial and attribute data were collected from organizations like Plan and Budget Organization, Water resources organization, Municipality and Ministry of roads and Urban development. Then, data were prepared to enter a GIS software environment. Figure 3 shows the main criteria and sub-criteria related to the problem.

It is notably to say, all layers are prepared in the city limit in detailed plans because this limit is the allowed limit for the city development in urban plans.
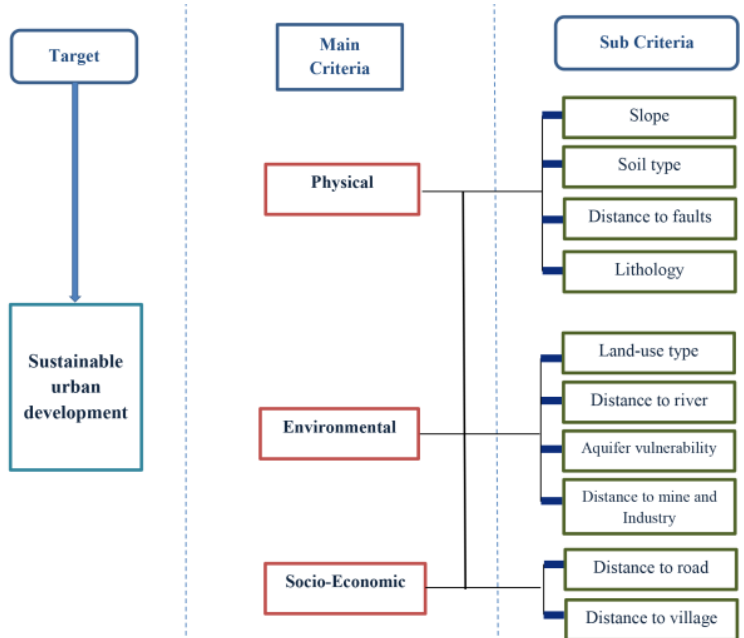

Figure 3. The influencing criteria and sub-criteria in location finding problem of the future city developments of Zanjan.

Table 1. Constraints and their corresponding values for subcriteria (Class 1 is the best value and Class 4 shows the worst),

The unit of slope is percentage and the distances in meter.

\begin{tabular}{|c|c|c|c|c|c|}
\hline Sub Criteria & Criteria & Class1 & Class2 & Class3 & Class4 \\
\hline Slope & $1 \%<\mathrm{S}<30 \%$ & $1 \%-5 \%$ & $5 \%-12 \%$ & $12 \%-20 \%$ & $20 \%-30 \%$ \\
\hline Distance to fault & $\mathrm{d}>300$ & $>1500$ & $1000-1500$ & $500-1000$ & $300-500$ \\
\hline Distance to river & $\mathrm{d}>50$ & $>1000$ & $500-1000$ & $300-500$ & $150-300$ \\
\hline Distance to industry & $\mathrm{d}>500$ & $>2000$ & $1500-2000$ & $1000-1500$ & $500-1000$ \\
\hline Distance to road & $\mathrm{d}>150$ & $>1000$ & $500-1000$ & $300-500$ & $150-300$ \\
\hline Distance to village & $\mathrm{d}>1500$ & $>5000$ & $3000-5000$ & $2000-3000$ & $1500-2000$ \\
\hline
\end{tabular}

\subsection{Criteria}


In this section, we will discuss each criterion and its priority in the allocation of urban land-use in the study area. It is notably to say, all the factor maps (figure 4 to 11 ) are created in UTM projection system based on WGS-84 datum.

\subsubsection{Slope}

One of the most important factors in the physical development of cities and construction of buildings is the slope of region (Sakieh et al, 2015). The most appropriate slope value for urban constructions is between $1 \%$ and $5 \%$. Areas with a slope of less than $1 \%$ are in difficulty in wastewater treatment and surface water, and on the other hand, a slope of over $30 \%$ will face serious problems in urban construction. The slope map of the study area is shown in Figure 4.

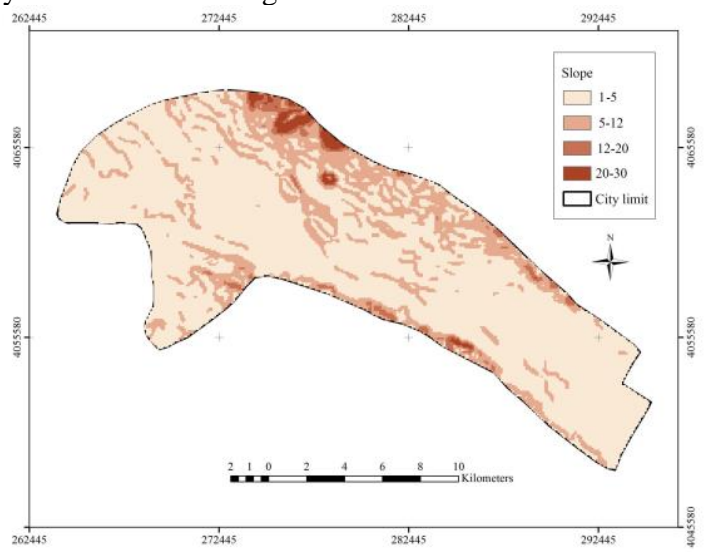

Figure 4. The slope map of the study area in terms of slope percentage

\subsubsection{Soil type}

The most important objective in soil studies in urban planning is the study of agricultural potential, and physical and mechanical properties of soil (Smidt et al., 2018). Figure 5 shows the soil type map in the study area considering FAO Soil Units (1974). The suitability of soil classes is shown in Figure 5 by the arrangement of categories in the legend. The Clacaric Regosols (Rc) is the best and the suitability degree decreases respectively. This classification is based on soil resistance for urban constructions.

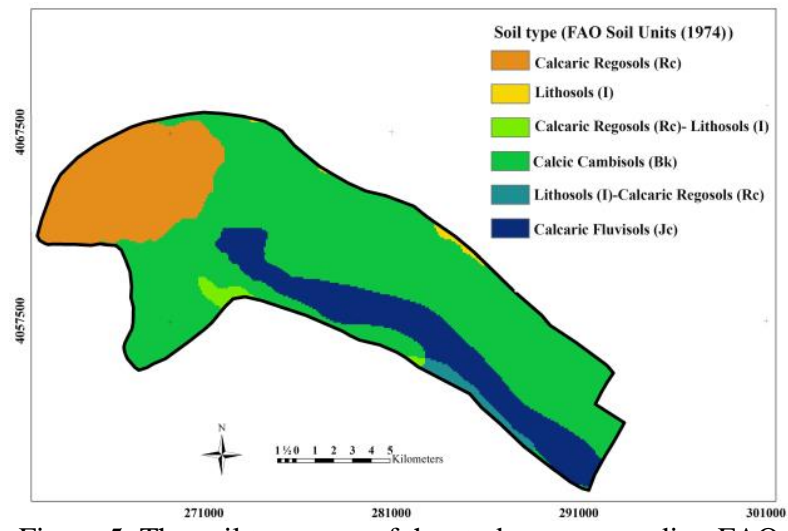

Figure 5. The soil type map of the study area according FAO soil units (1974)

\subsubsection{Lithology}

In land use planning geological studies is important from the standpoint of recognizing the resistant and bearing capacities (Liu et al., 2014). Figure 6 shows a map from the lithology perspective of the study area. Most of the Zanjan outcrops are composed of Eocene pyroclastic rocks (Karaj formation) (Khamechiyan et al., 2011). Figure 6 is obtained from the 1:100000 geology maps of Geological Survey of Iran. The suitability of Lithology classes also is shown in the legend of Figure 6 by the arrangement of categories. The Eocene granodiorite is the best and the suitability degree decreases respectively.

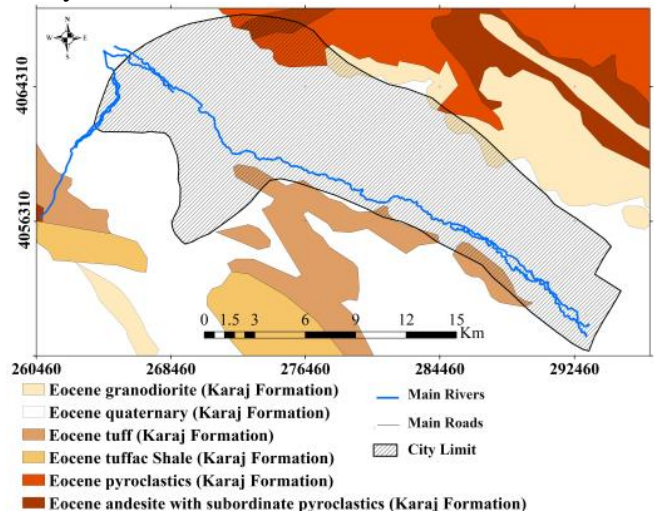

\subsubsection{Faults}

Figure 6 . The lithology map of the study area

Faults and their hazardous zones should be considered in city development (Bathtellos et al, 2017). The most dangerous building sites are the ones on top of faults, as seismic sources, and soft soils that amplifying the ground motion in earthquake (Foti et al., 2014). Therefore, when constructing residential areas, such areas should be avoided as far as possible, or at most, low density buildings should be built. Figure 7 shows the map of faults of the study area. The shown faults are derived from Zali et al. (2015).

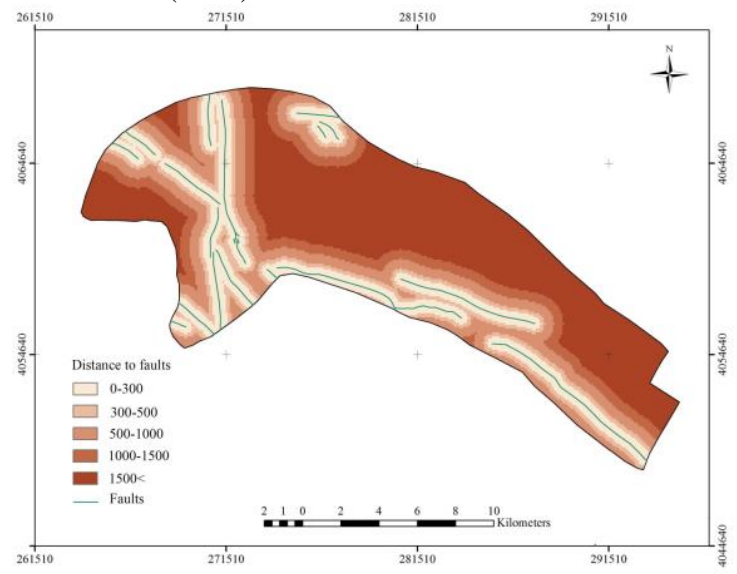

Figure 7. The existing faults in the study area and their hazardous zones (The unit of distance is meter)

\subsubsection{Land use}

Since the lands around cities may be agriculturally fertile, it is necessary to identify quantitative and qualitative features of these lands to prevent from construction for urban spatial development (Misra and Sharma, 2015). Figure 8 shows the land use map of the study area.

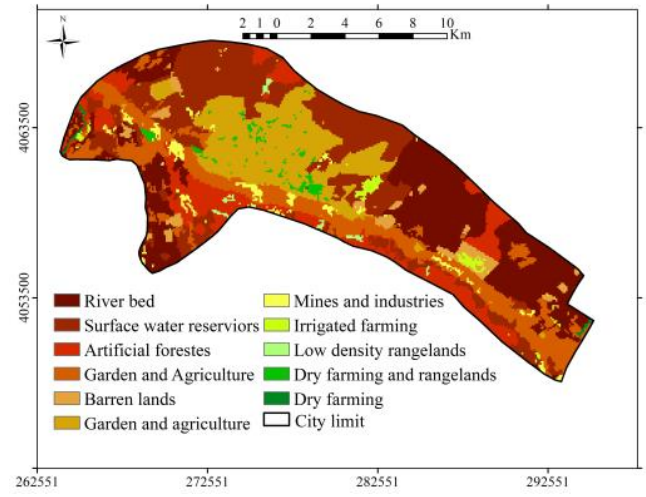


Figure 8- The land use map of the study area, The colour of units show the suitability of them for urban land use (The brown unit is the worst and the dark green unit shows the best lands)

According to the land use map of Zanjan, a large part of the area around the city is covered with high quality lands. However, agricultural lands continue along the Zanjanrood River.

\subsubsection{Distance to river}

In land use planning, it is necessary to identify the rivers and their limits to ensure that the flood does not threaten the buildings. Also, contamination of surface water due to urban activities should be considered (Chen, 2017). The most important source of surface water in Zanjan is the Zanjanrood river. In accordance with the rules concerning the bedding and limit of the rivers of the Ministry of Energy, this limit is in the range of 1 to 20 meters, and for the purpose of preserving the quality of water, it is up to 150 meters. Figure 9 shows the rivers and classified distances from them.

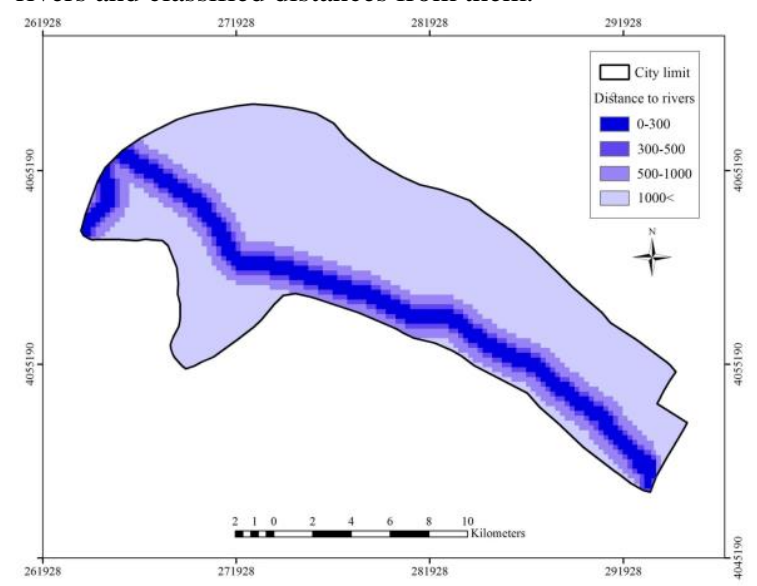

Figure 9. The map of main rivers in the study area and classified distances from them (The unit of distance is meter)

\subsubsection{Aquifer vulnerability}

The study of the vulnerability of groundwater is one of the environmental factors that should be considered in the physical development of cities (Sahoo et al., 2016). The city cannot develop in places where the vulnerability of underground water is high, because there is a possibility of pollution of underground water. Also, one of the important sources of water supply in Zanjan is groundwater, which may be prone to pollution from urban activities. Figure 10 shows the vulnerability map of Zanjan aquifer.

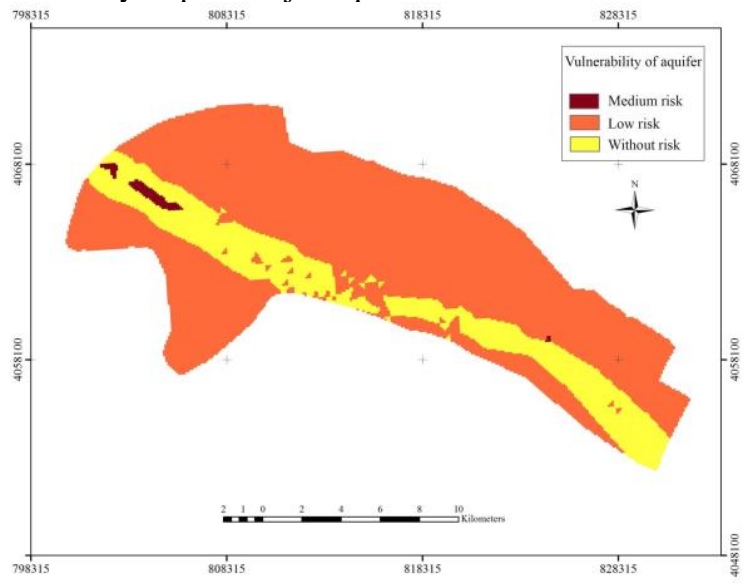

Figure 10. The vulnerability map of aquifer in the study area (Parizangeneh et al. 2014)
The aquifer vulnerability was obtained through the DRASTIC model by Parizangeneh et al. 2014. The acronym DRASTIC stands for the seven parameters which are Depth to water, net Recharge, Aquifer media, Soil media, Topography, Impact of vadose zone, and hydraulic Conductivity. The model yields a numerical index that is derived from weights assigned to the seven parameters (Taniguchi and Hiyama, 2014).

\subsubsection{Distance from mines and industrial zones}

Factories and industrial estates play an important role in noise and air pollution, and soil and water contamination. In order to locate suitable lands for urban development, it is important to observe the appropriate distance from the industrial areas (Martinat et al, 2016). Considering the dispersion of industrial and mining areas around case study area and according to the documents in the Environmental Protection Agency, a minimum distance of 500 meters from the industrial and mining zones should be respected for urban development.

\subsubsection{Distance from major roads and railways}

According to the rules of the Supreme Council for Urban Development and Architecture (Tarh \& manzar Consulting engineers, 2011), in order to respect the limit of roads and to prevent the development of cities towards roads, the construction of any building and facility at the distance of 150 meters of is prohibited. Then, it is considered as constraint in this research. Furthermore, when a city is near to the main roads of the national road network, some transformations and relations between cities have been effect on economic of region. So, these two issues should be considered in this factor.

\subsubsection{Distance from villages}

In addition to natural population growth in each city, other nonnatural factors such as the integration of villages with the city are involved in increasing the population of cities (Dahiya et al, 2018). On the other hand, as the increase in the active population of the city leads to rise of unemployment rate, social and economic problems in cities may eventually increase (Zaina et al., 2016). Therefore, lands with more than 1.5 kilometers distance of the villages around the city of Zanjan are considered for prospective urban development (Tarh \& manzar Consulting engineers, 2011). Figure 11 shows the villages in the study area and the classified distances from them.

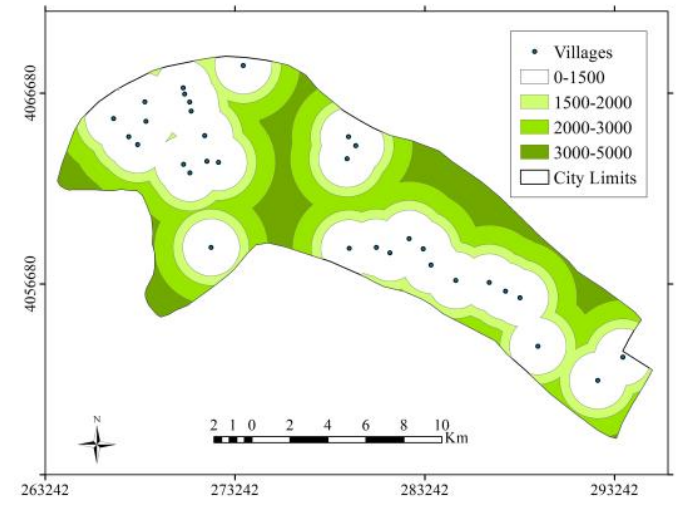

Figure 11. The map of existing villages in the study area and the distance map of villages (The unit of distance is meter)

\subsection{Weight assignment using AHP method}

After determining the effective criteria, in the next step, the evaluation of the layer was done using the opinions of 10 experts. The following steps have been taken to obtain the relevant weights (Saaty and Vargas, 2012).

- Paired comparison of the alternatives using the designed questionnaires according to AHP's common questionnaires,

- Creating a comparison matrix,

- Standardizing the matrix values; and ultimately, 
- And examining the degree of compatibility and finalizing the weight values.

The Readers can refer to Saaty and Vargas, 2012 for more details about AHP.

\subsection{Implementation}

In this section, the implementation of TOPSIS method for this study will be addressed. The details of the steps are as follows.

- Obtaining the decision matrix: the decision matrix consisting of the $m$ alternatives, i.e. the pixels under consideration, and the $n$ criterion, i.e. the layers corresponding to each criterion, is created. A sample of such a matrix is given in Equation 1.

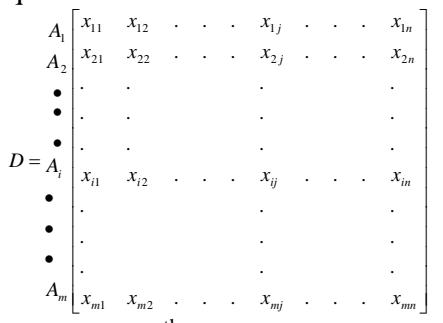

where $A_{i}$ is the $i^{\text {th }}$ pixel, and $x_{i j}$ is the value obtained from pixel $i^{\text {th }}$ of layer $j^{\text {th }}$.

- Quantifying and normalizing the decision matrix: the values of decision matrix are normalized using Equation 2.

$$
r_{i j}=\frac{x_{i j}}{\sqrt{\sum_{i=1}^{m} x_{i j}}} \quad j=1,2, \ldots, n, i=1,2, \ldots, m
$$

where $r_{i j}$ is the normalized value for each pixel.

- Weighting: At this stage, the weight that was determined for each criterion via the AHP approach is entered into the calculations, in the way that the weight of each criterion is multiplied in the normalized layer. In fact, the matrix $(V)$ is the product of the standard values of each criterion in its own weights.

$$
V_{i j}=\left[\begin{array}{cccc}
w_{1} r_{11} & w_{2} r_{12} & \ldots & w_{n} r_{1 n} \\
w_{2} r_{21} & w_{2} r_{22} & & w_{n} r_{2 n} \\
\cdot & \cdot & \cdot & \cdot \\
\cdot & \cdot & \cdot & \cdot \\
\cdot & \cdot & \cdot & \cdot \\
w_{1} r_{m 1} & w_{2} r_{m 2} & \ldots & w_{n} r_{m n}
\end{array}\right] \text { or } V_{i j}=w_{i} *_{i j}
$$

where $w_{i} \mathrm{~s}$ are weights obtained from the AHP for each criterion.

- Determining the positive ideal and the worst solutions: depending on the type of criterion and its effect on the objectives of decision-making, the positive ideal and the most negative alternatives are to be determined. These alternatives can be calculated using Equation (4).

$$
\begin{aligned}
& A^{+}=\left\{\left(\max _{i} v_{i j} \mid j \in J\right),\left(\underset{i}{\min } \mid j \in J^{\prime}\right) \quad i=1,2, \ldots, m\right\}=\left\{v_{1}^{+}, v_{2}^{+}, \ldots, v_{n}^{+}\right\} \\
& A^{-}=\left\{\left(\min _{i} v_{i j} \mid j \in J\right),\left(\max _{i} \mid j \in J^{\prime}\right) \quad i=1,2, \ldots, m\right\}=\left\{v_{1}^{-}, v_{2}^{-}, \ldots, v_{n}^{-}\right\}
\end{aligned}
$$

where $j$ and $j$ ' correspond to the criteria with positive and negative effects, respectively. where $v_{i j}$ ' is an element of the weighted matrix, and $v_{i}{ }^{+}$and $v_{i}{ }^{-}$are the positive ideal and the worst solutions, respectively.

- Calculating the distance of each alternative to the best and worst solutions using Equation 5.

$$
\begin{array}{ll}
d_{i}^{+}=\sqrt{\sum_{j=1}^{n}\left(v_{i j}-v_{j}^{+}\right)^{2}} & i=1,2, \ldots, m \\
d_{i}^{-}=\sqrt{\sum_{j=1}^{n}\left(v_{i j}-v_{j}^{-}\right)^{2}} & i=1,2, \ldots, m
\end{array}
$$

- Determining the closeness of the alternatives to the ideal solutions: After finding the distances of each alternative, the closeness of the solutions to the ideal state is determined using Equation 6.

$$
C L_{i}=\frac{d_{i}^{-}}{d_{i}^{-}+d_{i}^{+}}
$$

where $C L_{i}$ is the closeness value for the $i^{\text {th }}$ pixel relative to the worst solution. These values are in a range between 0 and $1 . C L$ values are calculated for each pixel in the study area.

\section{RESULTS}

\subsection{Results obtained from TOPSIS method}

After applying the weights (shown in Table 2) and implementing the TOPSIS method, the resulting map is shown in Figure 12. As seen in Figure 12, parts of the northwest of the city as well as the east and northeast parts are suitable for physical development. Also, some parts of the southwest after crossing Zanjanrood bed are suitable for development. In contrast, the western and southern parts of the study area are very unsuitable for the urban development. Also, of the total area under consideration, $6.9 \mathrm{~km}^{2}(2 \%)$ are in very low suitability class, $49.5 \mathrm{~km}^{2}(20 \%)$ in low suitability class, 159 $\mathrm{km}^{2}(66 \%)$ in moderate suitability, $22 \mathrm{~km}^{2}(10 \%)$ in high suitability, and $2.3 \mathrm{~km}^{2}(1.5 \%)$ in very high suitability.

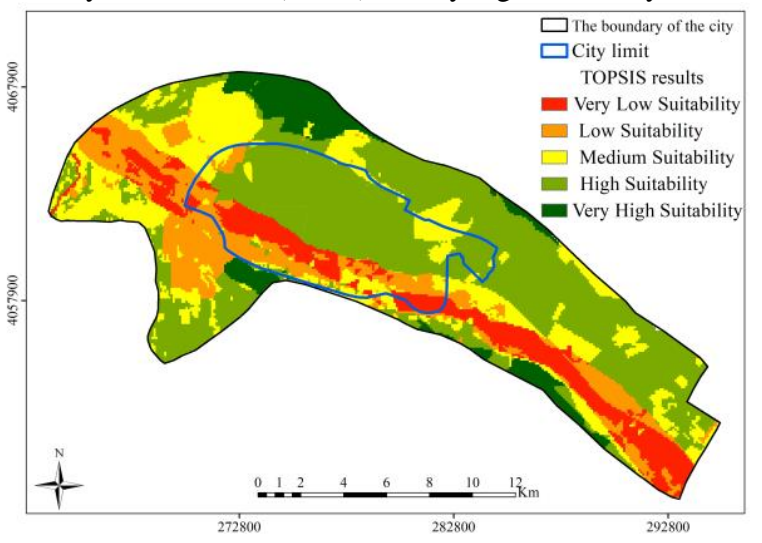

Figure 12. Classification of land suitability for physical development of the city using TOPSIS method based on $C L$ Figure 13 shows the urban boundary in the years 2005 and 2017. These boundaries are extracted using Landsat satellite images. As you can see, the main development direction of the city has been towards east, which in comparison with the results of this research is appropriate. On the other hand, some changes are seen towards west. As the development of the city toward west has been identified as undesirable in this study, it is necessary to consider some decisions in order to prevent from further in this direction. In total, about 15 percent of the city's development has been occurred on lands with low suitability.

Table 2. Final weights of sub-criteria based on the AHP method

\begin{tabular}{|l|c|c|}
\hline Criteria & Sub-Criteria & Weights \\
\hline Physical & Slope & 0.112 \\
& Soil & 0.097 \\
& Lithology & 0.112 \\
Environmental & Distance to fault & 0.142 \\
& Land-use & 0.045 \\
& Distance to river & 0.094 \\
& Aquifer vulnerability & 0.156 \\
Socio-economic & Distance to mine and Industry & 0.103 \\
& Distance to road & 0.083 \\
Sum & Distance to village & 0.054 \\
\hline
\end{tabular}




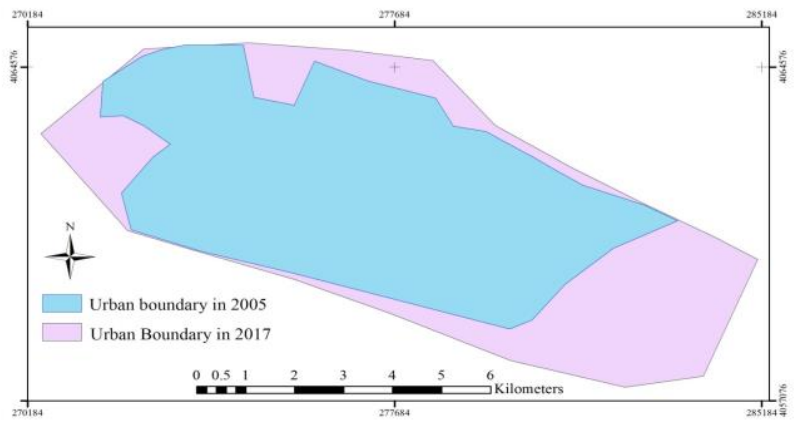

Figure 13. Comparison of the boundary of Zanjan city from 2005 through 2017

\subsection{Evaluation and discussion}

In order to investigate the efficiency of the TOPSIS method, it has been compared with the WSM method, one of the most common MCDM methods. In the WSM method, the suitability of each pixel is determined by using Equation 7. The results of this method are presented in Figure 14

$$
S_{i}=\sum_{i=1}^{n} W_{i} C_{i}
$$

where $S_{i j}$ is the final suitability of pixels for being an urban land use, $W_{i}$ is the weight of the $i^{\text {th }}$ criterion (in this research obtained from the AHP method), and $C_{i}$ is the value of that criteria in the layer under consideration. $S$ values have been standardised between 0 to 1 similar to $C L$ values.

By comparing Figures 12 and 14, it can be said that generally the areas in both models are close to each other for urban development. In order to more accurately compare two methods, of each main criterion, some sub-criterion with the highest weight was chosen, then the allocated area to urban land-use in 5 different suitability classes are computed. For example, from the physical criterion, the sub-criterion of distance to fault has the highest weight, which means it is the first layer to be studied. Table 3 shows the results of associated area for each suitability classes using TOPSIS and WSM methods for this sub-criterion. As seen in Table 3, in the extreme class (very high and very low) the TOPSIS method allocates less area than WSM. However, in the WSM method, even in areas with a low and very low suitability, some areas have been allocated for urban land use.

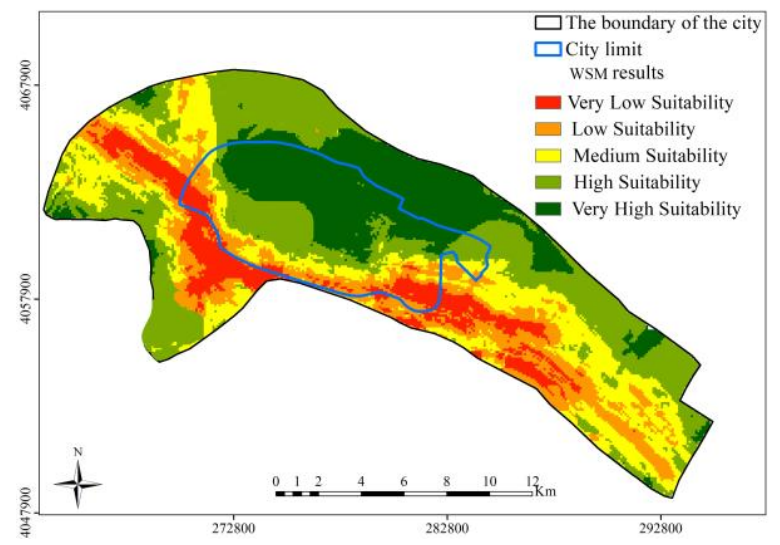

Figure 14. Classification of land suitability for physical development of Zanjan using WSM method based on normalized $S$ in Eq.7

Table 3. The results of the TOPSIS and WSM methods in the case of the sub-criterion of distance from fault

\begin{tabular}{|c|c|c|c|c|c|}
\hline \multirow{2}{*}{$\begin{array}{l}\text { E. } \\
\text { E. } \\
\text { 苞: } \\
\text { : }\end{array}$} & \multirow{2}{*}{ 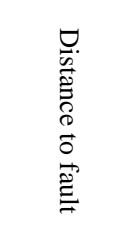 } & \multicolumn{2}{|c|}{ TOPSIS method } & \multicolumn{2}{|c|}{ WSM method } \\
\hline & & 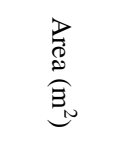 & 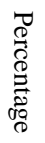 & 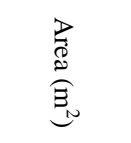 & 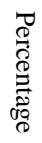 \\
\hline Very high & $>1500$ & 8837043 & 29 & 14928708 & 49 \\
\hline High & $1000-1500$ & 19432768 & 64 & 2491022.3 & 15 \\
\hline Medium & $500-1000$ & 2051476 & 6 & 4830063.1 & 8 \\
\hline Low & $300-500$ & 0 & 0 & 2735185.1 & 17 \\
\hline Very low & $0-300$ & 0 & 0 & 5417903.1 & 8 \\
\hline
\end{tabular}

Table 4 also shows the extent of the allocations in terms of the area in the low risk, medium risk, and vulnerable areas for the aquifer risk criterion. This criterion is also the most important criterion in the category of environmental criteria. Table 4 also shows that, in the TOPSIS method, areas with high risk of aquifer vulnerability are all categorized in the very low suitability. Also, most of the areas have been assigned to lands in low risk areas of aquifer vulnerability. In contrast, in the WSM method, most areas allocated to urban land use belong to the medium-risk areas.

Table 4- The extent of the allocations in terms of the area in the low risk, medium risk, and vulnerable areas for the aquifer risk criterion

\begin{tabular}{|c|c|c|c|c|c|c|c|}
\hline \multirow{3}{*}{$\begin{array}{c}\text { Aquifer } \\
\text { vulnerability/ } \\
\text { Suitability }\end{array}$} & \multicolumn{7}{|c|}{ WS method } \\
\hline & \multicolumn{2}{|c|}{ Low risk } & \multicolumn{3}{|c|}{ Medium risk } & \multicolumn{2}{|c|}{ High risk } \\
\hline & 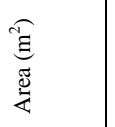 & 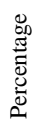 & \multicolumn{2}{|c|}{ 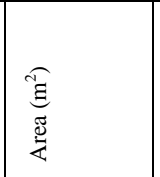 } & 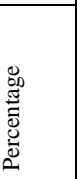 & \multirow[t]{2}{*}{ 急 } & \multirow{2}{*}{ 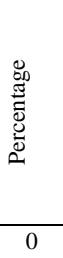 } \\
\hline Very high & 0 & 0 & \multicolumn{2}{|r|}{2539871} & 5 & & \\
\hline High & 31992 & 1 & \multicolumn{2}{|r|}{4837224} & 9 & 0 & 0 \\
\hline Medium & 20346 & 1 & \multicolumn{2}{|r|}{13946944} & 27 & 0 & 0 \\
\hline Low & 916347 & 52 & \multicolumn{2}{|r|}{18721186} & 37 & 700 & 0.09 \\
\hline Very low & 772778 & 44 & \multicolumn{2}{|r|}{10413367} & 21 & 772007 & 99.9 \\
\hline \multicolumn{8}{|c|}{ TOPSIS method } \\
\hline \multirow{2}{*}{$\begin{array}{c}\text { Aquifer } \\
\text { vulnerability/ } \\
\text { Suitability }\end{array}$} & \multicolumn{3}{|c|}{ Low risk } & \multicolumn{2}{|c|}{ Medium risk } & \multicolumn{2}{|c|}{ High risk } \\
\hline & 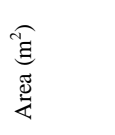 & & 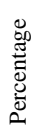 & 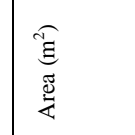 & 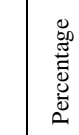 & 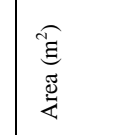 & 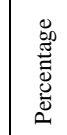 \\
\hline Very high & 0 & & 0 & 0 & 0 & 0 & 0 \\
\hline High & 8130760 & & 4 & 254304 & 1 & 0 & 0 \\
\hline Medium & 15496898 & & 82 & 4682356 & 9 & 0 & 0 \\
\hline Low & 22192681 & & 11 & 41188413 & 80 & 0 & 0 \\
\hline Very low & 2267511 & & 1 & 5071531 & 9 & 763452 & 100 \\
\hline
\end{tabular}

\section{CONCLUSIONS}

In the present study, the direction of sustainable development for future growths of Zanjan city was investigated. To this end, 
effective criteria for physical development were identified, and the required information and data from relevant organizations were collected and entered into GIS environment. Based on experts' opinions, priorities of the criteria were determined using the AHP method and then the TOPSIS model was used to identify the suitable areas for physical development of the city. The results show that the northwestern lands of Zanjan are in the highest priority, and the northeast, east and south parts of Zanjan, after the Zanjanrood River, are in the next priority. The lands of western Zanjan are in the third priority. Also, the comparison of the results of the TOPSIS and WSM methods showed that in the TOPSIS model ideal areas play important role in selecting suitable lands.

Since 2005 , about $15 \%$ of urban development has been carried out in unsuitable lands, requiring measures to be taken by the authorities. On the other hand, with considering more parameters in the model, especially social and economic parameters directly, modelling would be more accurate. Also, the introduction of decision-making policies would make the analysis more practical.

\section{REFERENCES}

Abdullahi, S., Pradhan, B., Mansor, S., Shariff, A. R. M. (2015). GIS-based modeling for the spatial measurement and evaluation of mixed land use development for a compact city. GISCI REMOTE SENS, 52(1), 18-39.

Bathrellos, G. D., Gaki-Papanastassiou, K., Skilodimou, H. D., Papanastassiou, D., Chousianitis, K. G. (2012). Potential suitability for urban planning and industry development using natural hazard maps and geological-geomorphological parameters. Environmental earth sciences, 66(2), 537-548.

Bathrellos, G. D., Skilodimou, H. D., Chousianitis, K., Youssef, A. M., Pradhan, B. (2017). Suitability estimation for urban development using multi-hazard assessment map. Sci Total Environ., 575, 119-134.

Chen, W. Y. (2017). Environmental externalities of urban river pollution and restoration: A hedonic analysis in Guangzhou (China). Landsc Urban Plan., 157, 170-179.

Dahiya, K., Sikarwar, S., Kumar, V. (2018). Structuring RuralUrban Integrated Growth, An Approach Towards Sustainable and Inclusive Regional Development: Case of Villages-Block Murthal, Dist. Sonipat, Haryana. Paper presented at the International Conference on Urban Sustainability: Emerging Trends, Themes, Concepts \& Practices (ICUS).

Delden, H., Hurkens, J. (2011). A generic Integrated Spatial Decision Support System for urban and regional planning Paper presented at the 19th International Congress on Modelling and Simulation, Perth, Australia.

Effat, H. A., Hegazy, M. N. (2013). A multidisciplinary approach to mapping potential urban development zones in Sinai Peninsula, Egypt using remote sensing and GIS. INT $J$ GEOGR INF SCI . 5(6), 567.

Liu, R., Zhang, K., Zhang, Z., Borthwick, A. G. (2014). Landuse suitability analysis for urban development in Beijing. $J$ ENVIRON MANAGE, 145, 170-179.

Martinat, S., Dvorak, P., Frantal, B., Klusacek, P., Kunc, J., Navratil, J., Reed, M. (2016). Sustainable urban development in a city affected by heavy industry and mining? Case study of brownfields in Karvina, Czech Republic. J Clean Prod. 118, 78-87.

Masoumi, Z., Maleki, J., Mesgari, M. S., Mansourian, A. (2017). Using an evolutionary algorithm in multiobjective geographic analysis for land use allocation and decision supporting. GEOGR ANN, 49(1), 58-83.

Misra, S. K., Sharma, S. (2015). Site Suitability Analysis for Urban Development: A Review. International Journal on
Recent and innovation Trends in Computing and Communication, 3(6), 3647-3651.

Phua, M. H., Minowa, M. (2005). A GIS-based multi-criteria decision making approach to forest conservation planning at a landscape scale: a case study in the Kinabalu Area, Sabah, Malaysia. Landsc Urban Plan., 71(2-4), 207-222.

Qin, X.-S., Huang, G. H., Chakma, A., Nie, X., Lin, Q. (2008). A MCDM-based expert system for climate-change impact assessment and adaptation planning-A case study for the Georgia Basin, Canada. Expert Syst Appl., 34(3), 2164-2179.

Saaty, T. L., Vargas, L. G. (2012). Models, methods, concepts applications of the analytic hierarchy process (Vol. 175): Springer Science \& Business Media.

Sahoo, M., Sahoo, S., Dhar, A., Pradhan, B. (2016). Effectiveness evaluation of objective and subjective weighting methods for aquifer vulnerability assessment in urban context. $J$ Hydrol., 541, 1303-1315.

Sakieh, Y., Amiri, B., Danekar, A., Feghhi, J., Dezhkam, S. (2015). Scenario-based evaluation of urban development sustainability: an integrative modeling approach to compromise between urbanization suitability index and landscape pattern. ENVIRON DEV SUSTAIN, 17(6), 1343-1365. doi:10.1007/s10668-014-9609-7

Santosh, C., Krishnaiah, C., Deshbhandari, P. G. (2018). Site suitability analysis for urban development using GIS based multicriteria evaluation technique: a case study in Chikodi Taluk, Belagavi District, Karnataka, India. Paper presented at the IOP Conference Series: Earth and Environmental Science. Schilder, M. (2016). Planning support systems in urban development in the Netherlands. (Master), Delft University of technology.

Shen, L. Y., Ochoa, J. J., Shah, M. N., Zhang, X. (2011). The application of urban sustainability indicators-A comparison between various practices. Habitat International, 35(1), 17-29. Smidt, S. J., Tayyebi, A., Kendall, A. D., Pijanowski, B. C., Hyndman, D. W. (2018). Agricultural implications of providing soil-based constraints on urban expansion: Land use forecasts to 2050. J ENVIRON MANAGE, 217, 677-689.

Tarh \& manzar Consulting engineers. (2011). Comprehensive Plan of Zarinabad City. Housing and Urban development organization Zanjan province.

Teriman, S., Yigitcanlar, T., Mayere, S. (2010). Sustainable urban development: an integrated framework for urban planning and development Rethinking sustainable development: urban management, engineering, and design (pp. 1-14): IGI Global.

Triantaphyllou, E. (2010). Multi-criteria Decision Making Methods: A Comparative Study. USA: Kluwer Academic Publisher.

Ullah, K. M., Mansourian, A. (2016). Evaluation of Land Suitability for Urban Land-Use Planning: Case Study Dhaka City. T GIS, 20(1), 20-37. doi:10.1111/tgis.12137.

Zaina, S., Zaina, S., Furlan, R. (2016). Urban planning in Qatar: strategies and vision for the development of transit villages in Doha. Australian Planner, 53(4), 286-301. 